\title{
Three Dimensional Structure Analysis of Cell Nuclei in Mice Cerebellar Cortex Using Array Tomography
}

\author{
M. Suga ${ }^{1,2}$, H. Nisioka ${ }^{1,2}$, M. Nakamura ${ }^{1}$, K. Suzuki ${ }^{1}$, K. Konishi ${ }^{3}$, T. Nonaka ${ }^{3}$, S. Kume ${ }^{2}$, M. Maeda $^{2}$, Y. \\ Kataoka $^{2}$, and K. Ohta ${ }^{4}$ \\ ${ }^{1}$ JEOL Ltd., Akishima, Tokyo, Japan \\ ${ }^{2}$ RIKEN CLST, Chuo-ku, Kobe, Hyogo, Japan \\ ${ }^{3}$ Nikon corporation, Sakae-ku, Yokohama, Kanagawa, Japan \\ ${ }^{4}$ Kurume University School of Medicine, Kurume, Fukuoka, Japan
}

Array tomography (AT) provides three-dimensional (3D) information by observing serial sections using scanning electron microscopy (SEM). Compared with serial block-face SEM and focused ion beam (FIB)/SEM, AT has several advantages: (a) High lateral resolution, (b) Standard staining with uranium and lead, allowing easy comparison of images captured by SEM and transmission electron microscopy (TEM), (c) Repetitive observations of samples, enabling hierarchical analysis from low magnification to high magnification, and (d) Low installation cost. In many cases, however, images of serial sections have been taken manually with labor and time. In addition, manual segmentation has been usually required. To solve these issues, we developed automatic image capturing software for AT with SEM. We also tried to use deep neural network to assist segmentation of the images.

In Figure 1, flow diagram of observation and analysis is shown. Mouse cerebellum cortex was fixed with glutaraldehyde and osmium tetra oxide, and was embedded in epoxy resin. Then serial sections were cut using ultra microtome and scooped on silicon substrate, and they were stained with uranyl acetate and lead citrate. SEM (JSM-7800F and JSM-7900F; JEOL Ltd.) was used for capturing images.

A software was developed for automatic capturing of SEM images in each serial section at the same position following manual identification of the corresponding apex of serial sections. Convolutional neural network algorithm was used to extract cell nuclei in each image. Since such an automatic segmentation was not perfectly completed, we manually corrected the data after the automatic segmentation. Then, 3D image was reconstructed by the stack of images from serial sections, and evaluated with quantitative analysis.

In Figure 2, images of cell nuclei in various cells are shown. Since the contrast of images was similar to that obtained by TEM, microstructures including cell nuclei were easily identified. A 3D-reconstructed image is shown in Figure 3. The automatic image capturing software and deep neural network analysis brought about easier procedure for the segmentation and quantitative analysis. Such a system for analysis of SEM images with AT will realize to be used for pathological diagnosis as well as basic biology.

References:

[1] Micheva et. al, Neuron, 55 (2007), p. 25. 

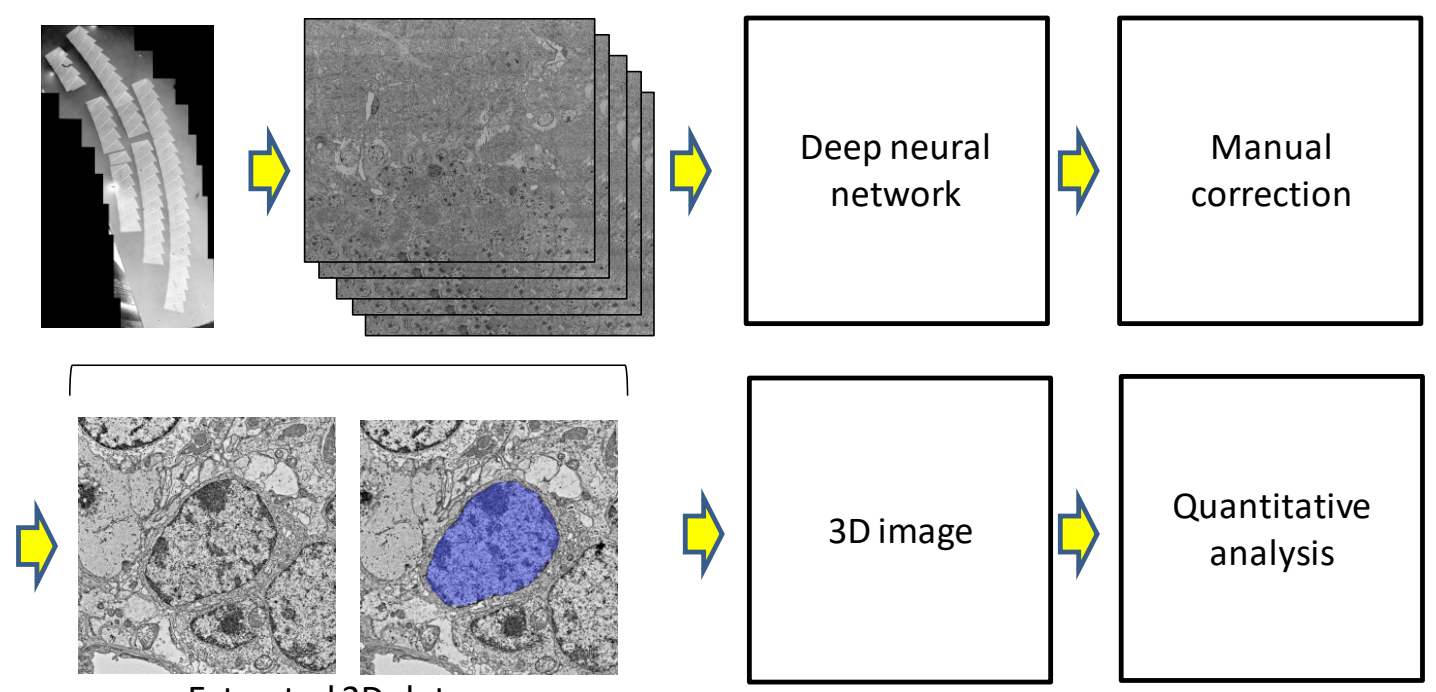

Figure 1. Flow diagram of Array Tomography and data analysis. Deep nerual netowrk was used to assist the feature extraction, segmentation, and data analysis.

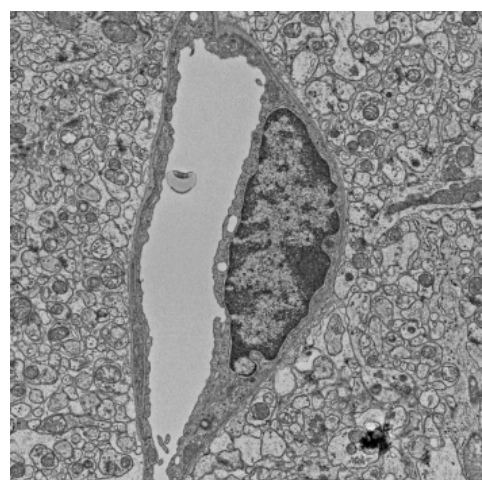

Endothelial cell

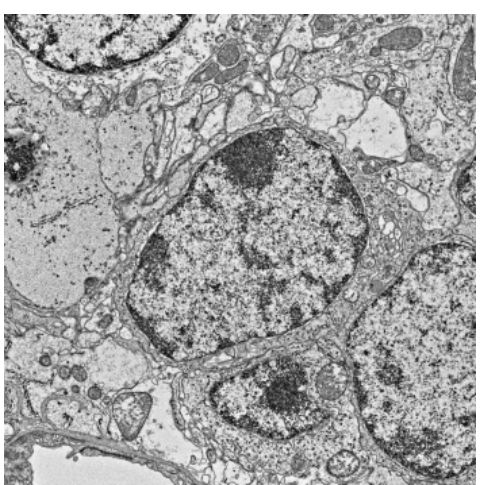

Granule cell

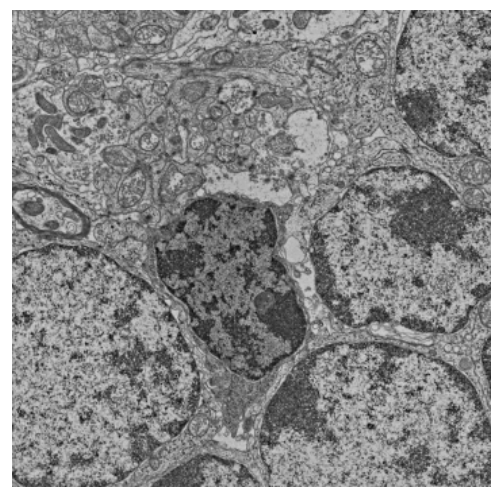

Microglia

Figure 2. Cell Nuclei of various cells were captured in each serial section. Since the contrast of images was similar to that obtained by TEM, microstructures including nuclei were easily identified.

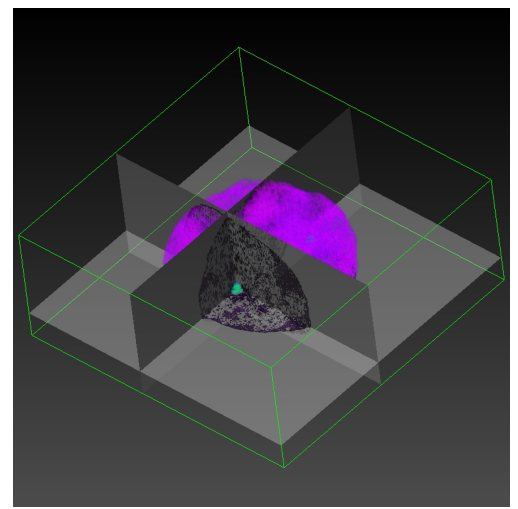

Figure 3. Reconstructed 3D data of a nucleus of granule cell. Purple indicates the surface of nucleus and green indicates nucleolus. 